https://doi.org/10.22319/rmcp.v11i2.4813

Nota de investigación

\title{
Análisis genético del desarrollo en peso vivo y tasa de gestación en primer parto en bovinos Brahman de Venezuela
}

\author{
Alejandro-Palacios-Espinosa ${ }^{\text {a }}$ \\ Omar-Verde ${ }^{b}$ \\ Narciso-Ysac-Ávila-Serrano ${ }^{c^{*}}$ \\ Alberto-Menéndez-Buxadera ${ }^{\mathrm{d}}$
}

a Universidad Autónoma de Baja California Sur, Departamento de Ciencia Animal y Conservación del Hábitat, La Paz, B.C.S., México.

b Unidad Territorial Yaracuy. Ministerio del Poder Popular para Ciencia, Tecnología e Innovación. Venezuela.

c Universidad del Mar, Cuerpo Académico "Ciencias Agropecuarias", Puerto Escondido, Oaxaca. México.

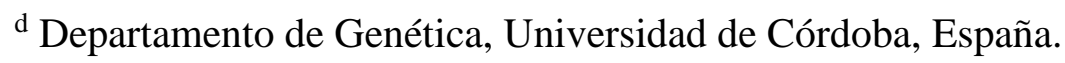

*Autor de correspondencia: reval1997@hotmail.com

\section{Resumen:}

El peso vivo (PV) de 2,777 animales (1,377 hembras y 1,400 machos con 53,258 datos individuales entre 30 y 600 días de edad), nacidos entre febrero de 2000 y junio del 2011, se analizaron con un modelo de regresión aleatoria (RA) para estimar los componentes genéticos de (co)varianza a lo largo de la escala edad-sexo. La tasa de gestación (TG) junto con el peso vivo ajustado a 548 días de edad (PA548) se estudiaron mediante un modelo multicaracter (MT) obteniéndose un incremento en los estimados de heredabilidad $\left(\mathrm{h}^{2}\right)$ para TG respecto al clásico modelo univariado $(0.08 \pm 0.03$ vs $0.11 \pm 0.02)$, aumentando la precisión del valor genético $(\mathrm{VG})$ para $\mathrm{TG}$ en $15.7 \%$. La correlación genética $\left(\mathrm{r}_{\mathrm{g}}\right)$ entre TG y PA548 fue $0.31 \pm 0.11$. El RA mostró que el PV a través de la edad no puede considerarse 
como expresión del mismo rasgo en ambos sexos, ya que las $r_{g}$ fueron inferiores a 0.60 . El análisis de componentes principales mostró que existen cambios importantes en la forma de crecimiento de los animales en la escala de edad representada en estos datos. Se manifestó un importante dimorfismo de origen genético, estimado como diferencia de los VG de machos y hembras en PV, el cual presenta una relación positiva con los VG de la TG.

Palabras clave: Heredabilidad, Correlaciones genéticas, Modelos multivariados, Regresión aleatoria, Dimorfismo sexual.

Recibido: $16 / 03 / 2018$

Aceptado: 21/03/2019

En la estación experimental 'La Cumaca' de la Facultad de Ciencias Veterinarias de la Universidad Central de Venezuela, se lleva a cabo un programa general de mejoramiento genético para características productivas de la raza Brahman, representando una importante fuente de genes para esta población ${ }^{(1)}$.

En la metodología aplicada para la realización de evaluaciones genéticas existen diferentes elementos críticos. En primer lugar, el PA548, con base en los cuales se estiman los valores genéticos (VG) de los animales puede estar sesgado, ya que se asume que el crecimiento es lineal. Resultados publicados ${ }^{(2,3,4,5)}$ en animales $B$. indicus, demuestran que existen variaciones en la forma de crecimiento a través de la trayectoria de las pruebas de comportamiento. En segundo lugar, generalmente el sexo del animal se considera como efecto fijo en el modelo, lo cual asume implícitamente que los componentes de (co)varianza son los mismos en ambos sexos. Este enfoque puede incorporar otra fuente de sesgo sobre las estimaciones de los VG, disminuyendo su precisión y por consiguiente, afectándose la marcha del programa de mejora ${ }^{(6,7,8)}$. Los elementos expuestos, pueden afectar las correlaciones genéticas del mismo rasgo entre ambos sexos $\left(\mathrm{r}_{\mathrm{HM}}\right)$, pudiéndose manifestar efectos de interacción genotipo-sexo.

El PV se ha expresado generalmente a un punto fijo, aunque parece muy lógico examinar estas relaciones a lo largo de la edad. Si la información está disponible, los componentes de (co)varianza pueden estimarse mediante modelos multivariados (MT) o, preferiblemente, por medio de regresión aleatoria (RA). Se han publicado resultados comparando modelos MT y RA para PV en ganado Bos indicus ${ }^{(2,4)}$, que demuestran las ventajas de RA. Sin embargo, en este enfoque longitudinal no se han examinado las relaciones entre los sexos de los animales. Por lo anterior, se requieren más evidencias, particularmente si se toma en cuenta posibles relaciones entre PV y comportamiento reproductivo (CR) de las hembras. 
Existe consenso sobre la importancia del CR para la economía del rebaño de carne. Sin embargo, la aparente baja $\mathrm{h}^{2}$ de la mayor parte de los rasgos reproductivos ${ }^{(9,10,11)}$ ha sido un factor limitante para su utilización como criterio de selección directa. Como alternativa, se han publicado resultados sobre la circunferencia escrotal (CE) o el PV medido en los machos jóvenes, y su respuesta correlacionada con el CR de las hembras medido por servicios por gestación; días al parto y TG al primer servicio. Aun cuando los resultados son alentadores ${ }^{(12,13)}$, estos se han expresado a una edad fija, manteniéndose la interrogante de la evolución de estas tendencias a lo largo de la edad hasta el primer parto.

Los objetivos de este estudio fueron: estimar las heredabilidades y las correlaciones genéticas entre el PA548 y la TG de las novillas en primera temporada de reproducción, mediante un MT; estimar los componentes de (co)varianza genética del PV de ambos sexos a lo largo de la edad mediante RA; comparar los valores genéticos para cada $i^{\text {th }}$ edad (VGi), estimados según RA, con los VG según MT.

La Estación Experimental La Cumaca, ubicada a 472 msnm cerca de la ciudad de San Felipe, estado Yaracuy, Venezuela, tiene una extensión de 433 ha, de las cuales 300 ha son cultivadas con pastos guinea, estrella, swazzi, pará y alambre. La precipitación anual promedio es de $1,650 \mathrm{~mm}$ con una temperatura promedio de 24 a $31.9^{\circ} \mathrm{C}$ y una humedad relativa promedio de $84 \%{ }^{(14)}$. Cuenta con un rebaño de ganado Brahman puro, registrado, de alrededor de 180 vacas en producción.

El peso vivo ajustado a 548 días de edad (PA548) de 3,120 animales nacidos entre febrero del 2000 y junio del 2011 se editó eliminando aquellos registros con incoherencias en el pedigrí, ausencia o problemas en la fecha de nacimiento. Finalmente, quedaron disponibles los registros de 2,777 animales (1,377 hembras y 1,400 machos). Estos animales eran hijos de 984 madres (729 en el vector de datos) y 107 sementales (48 en el vector de datos). El archivo de pedigrí incluyó 3,977 animales. Un total de 94,752 registros de PV individual, provenientes de 1,776 hembras y 1,864 machos, nacidos entre febrero del 1978 y junio del 2011 fueron utilizados. Estos animales eran hijos de 1,291 madres (929 en el vector de datos) y 128 sementales (58 en el vector de datos), y el archivo de pedigrí contó con 4,070 animales. Estos datos fueron editados, eliminado aquellos registros con incoherencias en el pedigrí, ausencia o problemas en la fecha de nacimiento y datos registrados a menos de 30 o más de 570 días de edad. Se eliminaron datos fuera del rango de \pm 3.2 desviaciones estándar dentro de clases de edades de 30 días de rango. Finalmente, quedaron disponibles un total de 53,258 datos individuales de 1,737 hembras y 1,803 machos.

Se corrieron varios modelos utilizando el procedimiento GLM de SAS ${ }^{(15)}$. El Cuadro 1 presenta algunos indicadores de los datos estudiados. 
Cuadro 1: Indicadores de peso vivo de animales Brahman en la estación experimental La Cumaca, Venezuela

\begin{tabular}{lcccc}
\hline Característica & $\begin{array}{c}\text { Tamaño de } \\
\text { muestra en } \\
\text { hembras }\end{array}$ & Hembras & $\begin{array}{c}\text { Tamaño de } \\
\text { muestra en } \\
\text { machos }\end{array}$ & Machos \\
\hline Peso al nacer & 1,776 & $29.5 \pm 4.5$ & 1864 & $31.9 \pm 4.9$ \\
Peso al destete & 1,639 & $165.8 \pm 26.2$ & 1630 & $177.3 \pm 28.3$ \\
Peso a 365 días & 1,396 & $209.9 \pm 29.8$ & 1410 & $231.8 \pm 34.9$ \\
Peso a 450 días & 1,378 & $235.6 \pm 32.0$ & 1392 & $271.2 \pm 38.9$ \\
Peso a 548 días & 1,340 & $290.0 \pm 34.1$ & 1385 & $326.3 \pm 42.2$ \\
Número de registros & 25,781 & 25781 & 27477 & 27477 \\
de peso vivo & 1,377 & $0.67 \pm 0.37$ & & \\
Tasa de Gestación & & & & \\
\hline
\end{tabular}

Tres bloques de análisis fueron realizados utilizando el programa Asreml3 ${ }^{(16)}$ :

Bloque 0. Modelo multivariado (MU) para PA548 y TG.

$$
\left[\begin{array}{l}
\mathbf{y}_{1} \\
\mathbf{y}_{2}
\end{array}\right]=\left[\begin{array}{cc}
\mathbf{X}_{1} & \mathbf{0} \\
\mathbf{0} & \mathbf{X}_{2}
\end{array}\right]\left[\begin{array}{l}
\mathbf{b}_{1} \\
\mathbf{b}_{2}
\end{array}\right]+\left[\begin{array}{cc}
\mathbf{Z}_{1} & \mathbf{0} \\
\mathbf{0} & \mathbf{Z}_{2}
\end{array}\right]\left[\begin{array}{l}
\mathbf{a}_{1} \\
\mathbf{a}_{2}
\end{array}\right]+\left[\begin{array}{l}
\mathbf{e}_{1} \\
\mathbf{e}_{2}
\end{array}\right]
$$

Donde:

$\mathbf{y i}_{\mathbf{i}} \quad$ es un vector correspondiente a PA548 y TG analizados al mismo tiempo;

$\mathbf{b}_{\mathrm{i}} \quad$ es un vector de efectos fijos de la $j k^{\text {th }}$ combinación del sexo-año-mes (con 275 niveles para PA548 y 124 para TG).

a es un vector aleatorio correlacionado debido al efecto genético aditivo del $i^{\text {th }}$ animal con datos y sus antecesores sin registros (4,070 niveles) para PA548 y TG;

eil es un vector residual aleatorio correlacionado entre los rasgos 1 y 2;

$\mathbf{X ~ y ~} \mathbf{Z}$ son matrices de incidencia que conectan los efectos fijos y aleatorios con el vector de observaciones.

En este modelo se asume que:

$$
\operatorname{Var}\left[\begin{array}{l}
\mathbf{a}_{\mathbf{i}} \\
\mathbf{e}_{\mathbf{i}}
\end{array}\right]=\left[\begin{array}{cc}
\mathbf{G}_{\mathbf{i}} & \mathbf{0} \\
\mathbf{0} & \mathbf{R}_{\mathbf{i}}
\end{array}\right]
$$




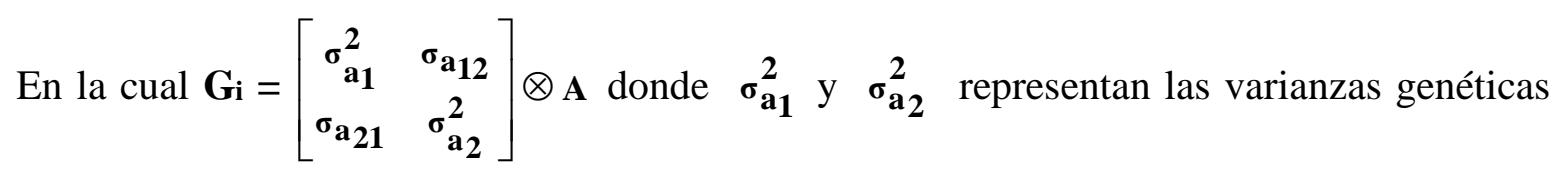
para ambos rasgos y $\boldsymbol{\sigma}_{\mathbf{a}_{\mathbf{1 2}}}$ su covarianza. Las (co)varianzas residual $\mathbf{R}_{\mathbf{i}}=\left[\begin{array}{cc}\boldsymbol{\sigma}_{\mathbf{e}_{\mathbf{1}}}^{2} & \boldsymbol{\sigma}_{\mathbf{e}_{\mathbf{1 2}}} \\ \boldsymbol{\sigma}_{\mathbf{e}_{\mathbf{2 1}}} & \boldsymbol{\sigma}_{\mathbf{e}_{2}}^{\mathbf{2}}\end{array}\right]$, contiene $\quad \boldsymbol{\sigma}_{\mathbf{e}_{\mathbf{1}}}^{\mathbf{2}}$ y $\quad \mathbf{\sigma}_{\mathbf{e}_{\mathbf{2}}}^{\mathbf{2}}$ que representan las varianzas para ambos rasgos y $\boldsymbol{\sigma}_{\mathbf{e}_{\mathbf{1 2}}}$ es su covarianza. A es la matriz de parentesco entre todos los animales y $\otimes$ es el símbolo de producto. Con estos parámetros se estimó la $\mathrm{h}^{2}$ para cada rasgo $\left(\mathbf{h}_{\mathbf{a}_{\mathbf{i}}}^{\mathbf{2}}\right)$ y las correlaciones genéticas $\left(\mathbf{r}_{\mathrm{gi}}\right)$, entre ambos caracteres, mediante funciones lineales de los correspondientes componentes y utilizando fórmulas clásicas ${ }^{(17)}$. Los VG para cada rasgo se estimaron como solución del modelo descrito, y la exactitud $\left(\mathrm{Acc}_{\mathrm{ij}}\right)$ de tales estimaciones según:

$$
\operatorname{Acc}_{\mathrm{ij}}=\sqrt{1-\frac{\mathbf{P e v}}{\sigma_{\mathrm{i}}^{2}}} * 100
$$

Donde: Pev es la varianza del error de predicción (valor propio para cada animal para el carácter estudiado), y $\sigma_{\mathbf{i}}^{2}$ : es la varianza genética del carácter en la población estudiada. Este mismo modelo fue aplicado en forma univariada, para presentar parámetros similares a los aplicados con el programa original de la Estación Experimental.

Para el análisis de PV a lo largo de la trayectoria de la edad se aplicó RA, utilizando varios modelos y sin considerar los efectos maternos, los cuales variaron en el orden de ajuste del polinomio para los efectos aleatorios, así como las estimaciones de componentes de (co)varianza total o intra sexo del animal. En total se realizaron dos bloques de modelos:

Bloque 1- Se asume que los componentes de (co)varianza son los mismos en ambos sexos.

$$
\begin{aligned}
& \mathbf{y}_{\mathbf{i j k l}}=\mathbf{f i x e d}_{\mathbf{i}}+\operatorname{sex}_{\mathbf{j}}+\sum_{\mathbf{r}=\mathbf{0}}^{3} \Phi_{\mathbf{r}} \mathbf{b}_{1 \mathbf{r}}+\sum_{\mathbf{r}=\mathbf{0}}^{1} \Phi_{\mathbf{r}} \mathbf{a}_{\mathbf{k r}}+\sum_{\mathbf{r}=\mathbf{0}}^{1} \Phi_{\mathbf{r}} \mathbf{p}_{\mathbf{i r}}+\mathbf{Z}_{1} \mathbf{q}_{\mathbf{m}}+\mathbf{R} \rightarrow \text { Model } 1 \\
& \mathbf{y}_{\mathbf{i j k l}}=\text { fixed }_{\mathbf{i}}+\operatorname{sex}_{\mathbf{j}}+\sum_{\mathbf{r}=0}^{3} \Phi_{\mathbf{r}} \mathbf{b}_{1 \mathbf{r}}+\sum_{\mathbf{r}=0}^{2} \Phi_{\mathbf{r}} \mathbf{a}_{\mathbf{k r}}+\sum_{\mathbf{r}=0}^{1} \Phi_{\mathbf{r}} \mathbf{p}_{\mathbf{i}_{\mathbf{r}}}+\mathbf{Z}_{1} \mathbf{q}_{\mathbf{m}}+\mathbf{R} \rightarrow \text { Model } 2 \\
& \mathbf{y}_{\mathbf{i j k l}}=\text { fixed }_{\mathbf{i}}+\operatorname{sex}_{\mathbf{j}}+\sum_{\mathbf{r}=\mathbf{0}}^{3} \Phi_{\mathbf{r}} \mathbf{b}_{1 \mathbf{r}}+\sum_{\mathbf{r}=\mathbf{0}}^{3} \Phi_{\mathbf{r}} \mathbf{a}_{\mathbf{k r}}+\sum_{\mathbf{r}=0}^{1} \Phi_{\mathbf{r}} \mathbf{p}_{\mathbf{i r}}+\mathbf{Z}_{1} \mathbf{q}_{\mathbf{m}}+\mathbf{R} \rightarrow \text { Model } 3
\end{aligned}
$$


Bloque 2- Se asume que los componentes de (co)varianza no son los mismos en ambos sexos.

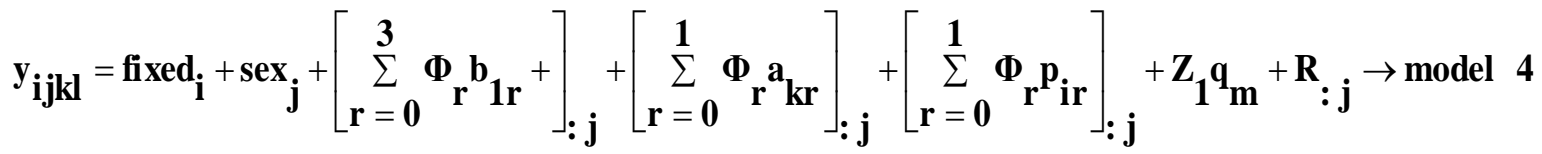

$$
\begin{aligned}
& \mathbf{y}_{\mathbf{i j k l}}=\mathbf{f i x e d}_{\mathbf{i}}+\mathbf{s e x}_{\mathbf{j}}+\left[\underset{\mathbf{r}=\mathbf{0}}{\sum_{\mathbf{r}}} \Phi_{\mathbf{r}} \mathbf{b}_{\mathbf{1 r}}+\right]_{: \mathbf{j}}+\left[\sum_{\mathbf{r}=\mathbf{0}}^{2} \Phi_{\mathbf{r}} \mathbf{a}_{\mathbf{k r}}\right]_{: \mathbf{j}}+\left[\underset{\mathbf{r}=\mathbf{0}}{1} \Phi_{\mathbf{r}} \mathbf{p}_{\mathbf{i r}}\right]_{: \mathbf{j}}+\mathbf{Z}_{\mathbf{1}} \mathbf{q}_{\mathbf{m}}+\mathbf{R}: \mathbf{j} \rightarrow \operatorname{model}^{5}
\end{aligned}
$$

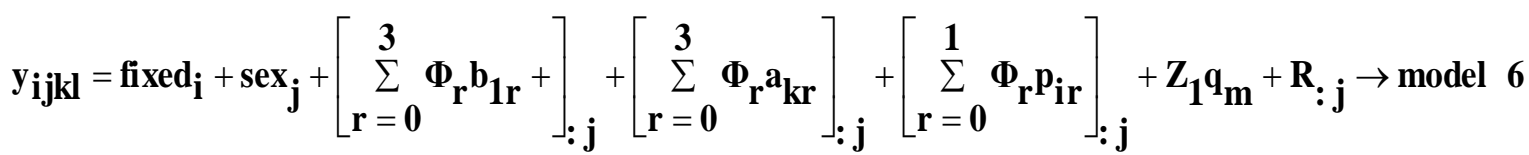

En ambos bloques $\mathrm{Y}_{\mathrm{ijkl}}$ representa las diferentes estimaciones de PV en el 1 ak ésimo animal, del j ésimo sexo. Los efectos fijos (fixedi) fueron año-mes de control con 674 niveles: sexoedad al parto de la vaca con 18 niveles representados en todos los modelos, de manera que los resultados pueden compararse aplicando los criterios informativos LogL; BIC y AIC. Los seis modelos solo se diferencian en el orden de ajuste del polinomio de Legendre ( $\boldsymbol{\Phi}_{\mathrm{i}}$ ) para los efectos aleatorios y la varianza residual $(\mathrm{R})$ que se considera homogénea para el bloque $1 \mathrm{e}$ intra $\mathrm{j}^{\text {th }}$ sexo en el bloque 2 . La estrategia aplicada en el bloque 2 fue la de hacer estimaciones de componentes de (co)varianza entre e intra sexo del animal. La matriz de incidencia $Z_{1}$ contiene como elementos 1 ó 0 para conectar cada observación con los efectos aleatorios de ambiente permanente maternal $\left(\mathrm{q}_{\mathrm{m}}\right)$ con 91 niveles. Para ambos bloques, la curva de crecimiento de la población fue modelada por un coeficiente de regresión $\left(b_{1}\right)$ de la edad sobre el peso vivo, mediante los coeficientes de $\boldsymbol{\Phi}_{\mathrm{i}}$ de orden 3 , mientras que fue de orden $\mathbf{r}=1,2,3$ para los efectos aleatorios genéticos y de orden 1 para el efecto permanente individual $\left(\mathrm{p}_{\mathrm{i}}\right)$, debido a repeticiones del mismo rasgo en los animales a lo largo de la escala de edades. Los componentes de varianza esperados en ambos bloques fueron los siguientes:

$$
\mathbf{y} \sim \mathbf{N}\left[\mathbf{0},\left(\boldsymbol{\sigma}_{\mathrm{y}}^{2}=\Phi_{\mathrm{i}} *\left[\mathbf{G}_{\mathbf{0}}=\left(\mathrm{A} \otimes \mathbf{K}_{\mathrm{G}}\right)\right]^{*} \Phi^{\prime}+\mathbf{I}_{\mathrm{p}} \boldsymbol{\sigma}_{\mathrm{i}}^{2}+\mathbf{I}_{\mathbf{q}} \boldsymbol{\sigma}_{\mathrm{m}}^{2}+\mathbf{I}_{\mathrm{n}} \boldsymbol{\sigma}_{\mathrm{e}}^{2}\right] \text { bloque } 1\right.
$$

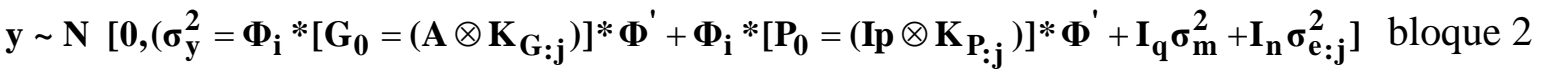

donde A es el numerador de la matriz de parentesco entre los animales con datos y sus antecesores sin registros ( $n=4,070$ animales totales). $I_{p}$ es la matriz de identidad para los efectos aleatorios de ambiente permanente individual (dimensión $\mathbf{p}=3540$ para el bloque 1 con varianza $\boldsymbol{\sigma}_{\mathbf{i}}^{2}, \mathrm{p}_{\mathrm{h}}=1,737 \mathrm{y} \mathrm{p}_{\mathrm{m}}=1,803$ niveles para las hembras y machos respectivamente en el bloque 2 con varianzas contenidas en la matriz de regresión aleatoria de efectos permanente individual intra $\mathbf{j}^{\text {th }}$ sexo del animal $\left(\mathbf{K}_{\mathbf{P}: \mathbf{j}}\right)$. $I_{\mathrm{q}}$ es la matriz de incidencia para ambiente permanente materno (dimensión $\mathbf{q}=1,291$ madres y varianza $\boldsymbol{\sigma}_{\mathbf{m}}^{\mathbf{2}}$ ). $\mathrm{R}$ es el error residual con $I_{n}$ como matriz de incidencia $\left(n=53,258\right.$ registros en el bloque 1 y varianza $\sigma_{e}^{2}$ y 
$\mathrm{n}_{\mathrm{h}}=25,781$ y $\mathrm{n}_{\mathrm{m}}=27,477$ para hembras y machos en el bloque 2 con varianza $\boldsymbol{\sigma}_{\mathbf{e}: \mathbf{j}}^{\mathbf{2}}$, respectivamente). $\mathrm{G}_{0}$ contienen una matriz de regresión aleatoria $\left(\mathrm{K}_{\mathrm{G}}\right)$ de dimensión $(\mathrm{r}+1)^{*}(\mathrm{r}+1)$, en los modelos más complejos del bloque 2 los elementos serán:

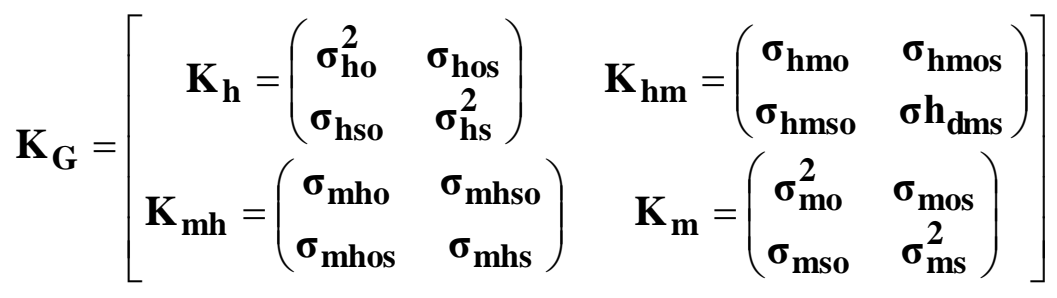

La versión empleada del asreml produce automáticamente el análisis de componentes principales de esta matriz, la cual facilita la interpretación de las trayectorias de las (co)varianzas estimadas. En este caso, $\mathrm{K}_{\mathrm{G}}$ es una matriz simétrica conformada por cuatro sub matrices con los mismos componentes que contiene las (co)varianzas para efectos genéticos en las hembras $\left(\mathrm{K}_{\mathrm{h}}\right)$; machos $\left(\mathrm{K}_{\mathrm{m}}\right)$ y sus covarianzas $\left(\mathrm{K}_{\mathrm{hm}}\right)$ con sus respectivas varianzas del intercepto $\left(\boldsymbol{\sigma}_{\text {ho }}^{2}\right.$ y $\left.\boldsymbol{\sigma}_{\text {mo }}^{2}\right)$; pendiente $\left(\boldsymbol{\sigma}_{\mathrm{hs}}^{2}\right.$ y $\left.\boldsymbol{\sigma}_{\mathrm{ms}}^{2}\right)$ y sus covarianzas $\left(\boldsymbol{\sigma}_{\mathrm{hso}} ; \boldsymbol{\sigma}_{\mathrm{mso}} ; \boldsymbol{\sigma}_{\mathrm{mhs}} \mathrm{y}\right.$ $\left.\boldsymbol{\sigma}_{\mathbf{h m o s}}\right)$. En estos casos los subíndices o y s indican intercepto y pendiente, respectivamente. Esta matriz descrita es aplicable a un orden de ajuste $r=1$. Es por ello por lo que cada sub matriz es de dimensión $2 \times 2$; para $r=2$ será de $3 \times 3$ y para orden $r=3$ será de $4 \times 4$ y los componentes adicionales serán los términos cuadrático y cúbico, respectivamente. Para el bloque 1 la matriz $\mathrm{K}_{\mathrm{G}}$ no presenta los estimados de cada sexo. Para ambos bloques, manipulando los elementos de estas matrices, así como los coeficientes del polinomio de Legendre $\left(\boldsymbol{\Phi}_{\mathbf{i}}\right)$ de orden r se pueden estimar los componentes de (co)varianza a lo largo de la trayectoria de edad y cada sexo ${ }^{(18)}: \sigma_{\mathbf{h i}}^{2}=\Phi_{i} K_{h} \Phi_{i}^{\prime} ; \sigma_{m i}^{2}=\Phi_{i} K_{m} \Phi_{i}^{\prime} \mathbf{y} \sigma_{h m_{i j}}=\Phi_{i} K_{h m} \Phi_{j}^{\prime}$.

En general, los parámetros genéticos de $\mathrm{h}^{2} \mathrm{y} \mathrm{r}_{\mathrm{g}}$ se estiman aplicando fórmulas clásicas ${ }^{(17)}$. Los estimados de VG del PV para cada sexo se estiman mediante la solución del mejor modelo donde, para el $\mathrm{k}^{\text {th }}$ animal se tendrá:

$$
\mathbf{V G}_{\mathbf{k}}^{\mathbf{i}}=\mathbf{a}_{\mathbf{k}} \boldsymbol{\Phi}_{\mathbf{i}}^{\prime}
$$

y donde $\Phi_{\mathbf{i}}$ son los correspondientes coeficientes del polinomio de Legendre para cada $i^{\text {th }}$ punto de la escala de edad. En este modelo, cada animal (total; hembra o macho) estará expresado por una función genética $\left(\mathrm{a}_{\mathrm{k}}\right)$ vinculada a los efectos del intercepto, pendiente y otros términos de acuerdo al orden de ajuste del polinomio seleccionado.

Los parámetros genéticos resultantes del bloque 0 se presentan en el Cuadro 2, donde se incorporaron las correlaciones entre los VG estimados por MU y MT. 
Cuadro 2: Componentes de (co)varianza y heredabilidad del peso vivo ajustado a 548 días y la tasa de gestación al primer parto (Modelos del bloque 0)

\begin{tabular}{lcc}
\hline Parámetro genético & Peso vivo (kg) & $\begin{array}{c}\text { Tasa de gestación } \\
(\mathbf{d})\end{array}$ \\
\hline Varianza genética-MU & 369.9 & 0.017588 \\
Varianza genética-MT & 375.3 & 0.02335 \\
Heredabilidad- MU & $0.337 \pm 0.11$ & $0.084 \pm 0.03$ \\
Heredabilidad- MT & $0.349 \pm 0.10$ & $0.109 \pm 0.02$ \\
Corr genética- Peso y tasa de gestación & \multicolumn{2}{c}{$0.309 \pm 0.11$} \\
Precisión, \% del valor genético (VG) MU & $63.5 \pm 12.1$ & $34.2 \pm 8.9$ \\
Precisión, \% del valor genético MT & $64.1 \pm 12.1$ & $39.6 \pm 9.8$ \\
Corr entre valores genéticos MU x MT & 0.996 & 0.8971 \\
Corr VG tasa de gestación MU x VG peso MU & & 0.286 \\
Corr VG gestación MU x VG peso MT & \multicolumn{2}{c}{0.570} \\
\hline
\end{tabular}

Para PA548 no hubo diferencias entre MU y MT. Para TG, el MT incrementó la h², mejorando las precisiones de los VG. La $r_{g}$ entre ambos caracteres fue positiva $\left(r_{g}=0.309\right)$, indicando ausencia de antagonismo en la mejora de los dos rasgos. Las correlaciones entre los VG según ambos modelos fueron superiores a 0.897, de lo que se infiere que no existirán cambios en el orden de mérito por ambos procedimientos. El modelo MT tiene ventajas adicionales, manifestadas en una mayor correlación con el VG para TG, así como mayor precisión en los estimados de VG para este último rasgo, cuyo valor de $\mathrm{h}^{2}$ fue bajo.

La bondad de ajuste de los seis modelos de los bloques 1 y 2 se determinó mediante los criterios LogL, AIC y BIC, los tres coincidieron en que el polinomio de tercer orden para el efecto genético es el de mejor ajuste a los datos. En particular, los modelos del bloque 2 presentan mejores resultados, que demuestran que existe una significativa variación entre sexos para los componentes genéticos de (co)varianza.

La $\mathrm{h}^{2}$ del PV a lo largo de la edad para ambos sexos, así como la correlación genética entre ellos se presentan en la Figura 1. Las tendencias de $\mathrm{h}^{2}$ presentan ligeros aumentos conforme avanza la edad, siendo superiores para las hembras. Las $r_{g}$ reflejan un patrón inverso con valores entre 0.25 a 0.35. Por otro lado, la distribución de frecuencia de los VG para PA548, estimados según el modelo del bloque 0 y según RA se muestran en la Figura 2, en donde puede observarse una superposición en los tres estimados de VG. El análisis de componentes principales de la matriz KG del modelo 6 seleccionado, indica que el primer (vp1) y segundo vector propio (vp2) explicaron el 57 y $31 \%$ de la variación genética, respectivamente. Los VG de los mejores 200 animales del MU (método oficial actualmente en uso) y de la RA a lo largo de la edad y para cada sexo, se muestran en la Figura 3. En ella se visualiza que la 
tendencia en todos los machos es positiva, mientras que en las hembras se pueden encontrar animales con VG negativos.

Figura 1: Estimados de heredabilidad y correlación genética para el peso vivo de hembras y machos a lo largo de la escala de edad en animales Brahman (modelo 6)

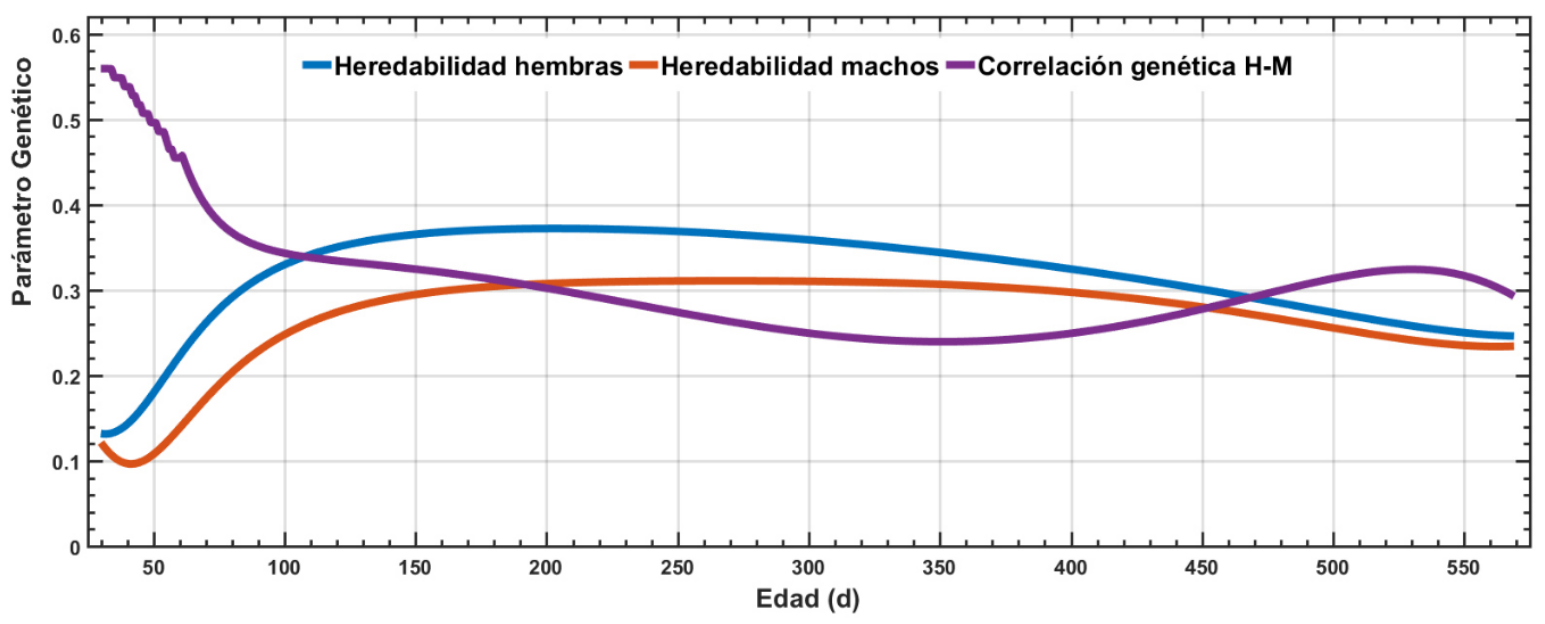

Figura 2: Distribución de frecuencia de los valores genéticos para peso a 548 días de edad según modelo actualmente en uso y mediante regresión aleatoria (modelo 6)

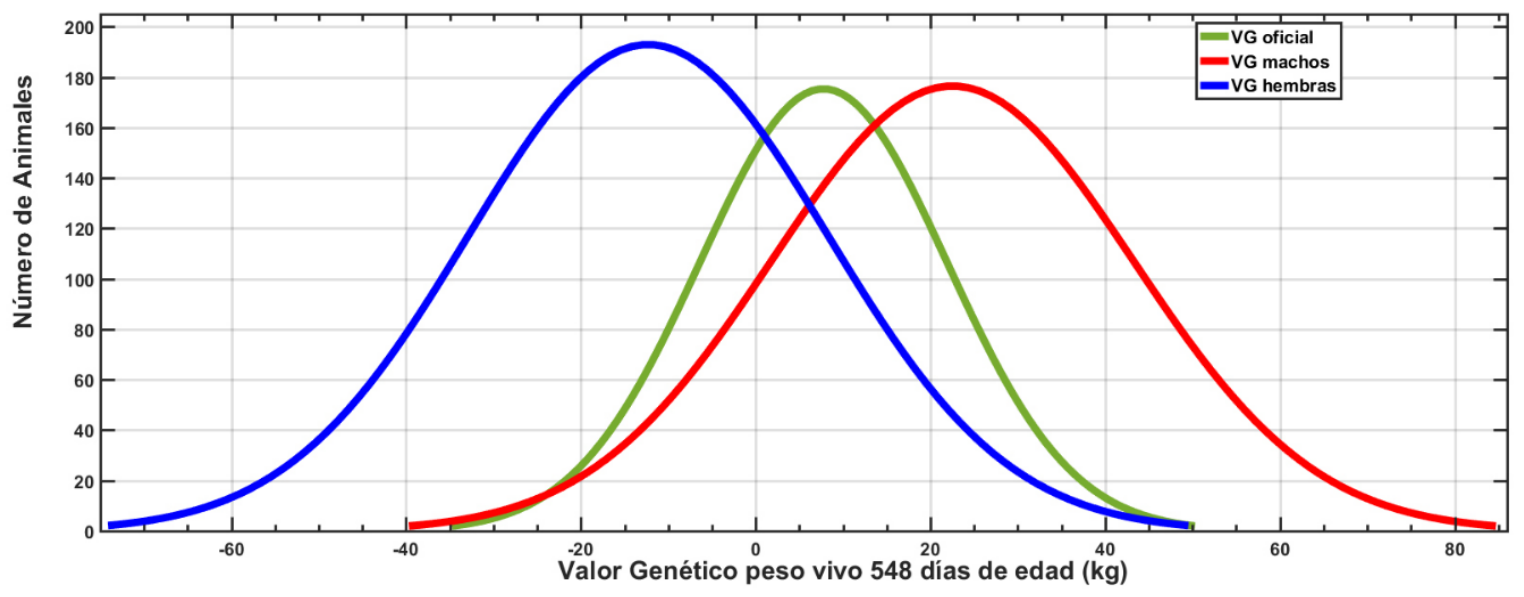


Figura 3: Evolución de los Valores genéticos de animales Brahman de cada sexo a lo largo de la trayectoria de edad, seleccionados según modelo actual de evaluación
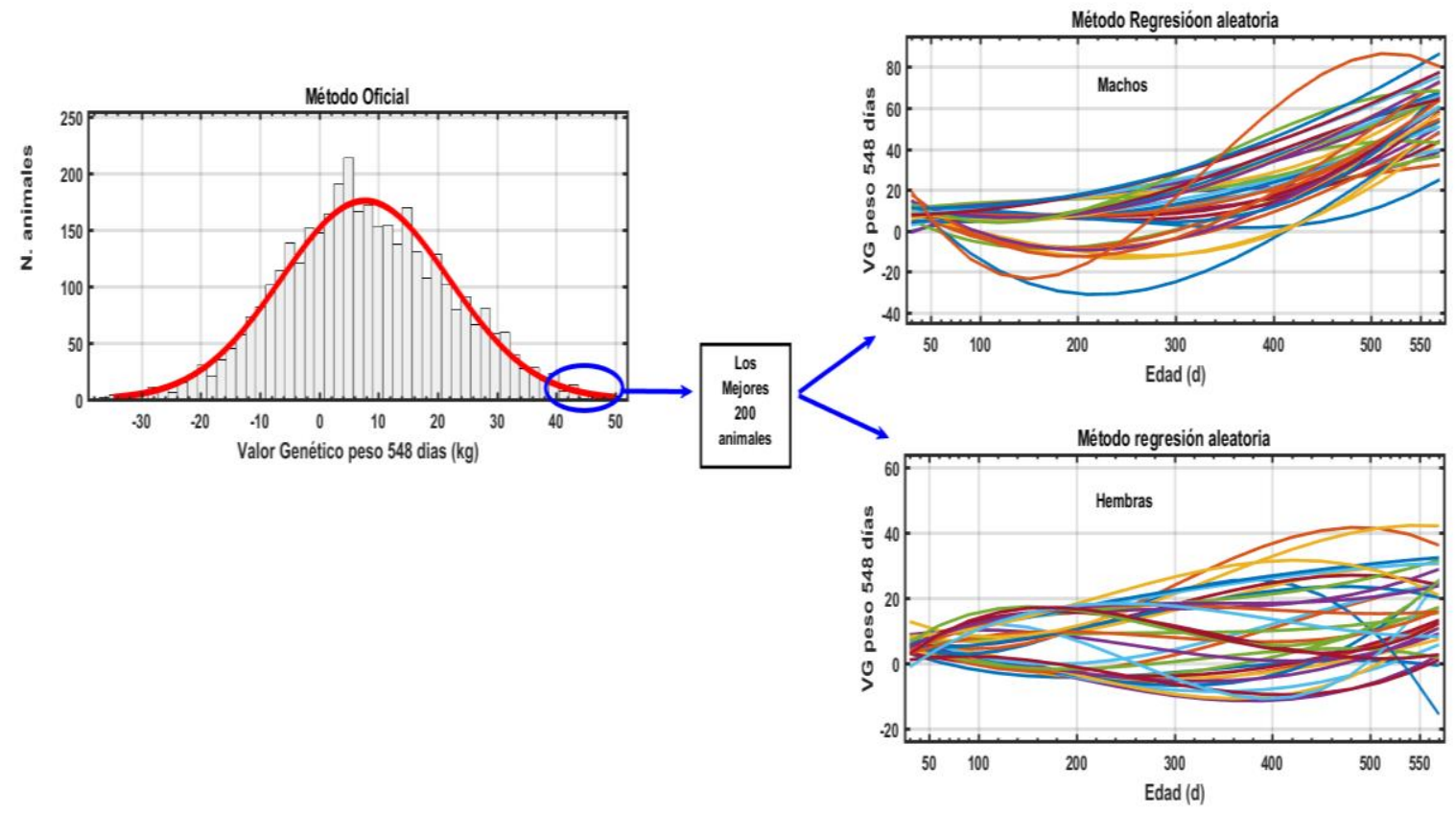

La evolución del mérito respecto al año de nacimiento de los animales se muestra en la Figura 4. El progreso genético anual fue de $0.933 \pm 0.021 \mathrm{~kg} / \mathrm{año}$ para PA548 el carácter principal en el programa de mejora aplicado, mientras que para TG fue de $0.354 \pm 0.010 \%$ /año.

Figura 4: Evolución del Mérito genético para peso vivo y tasa de gestación en animales Brahman de la estación experimental La Cumaca

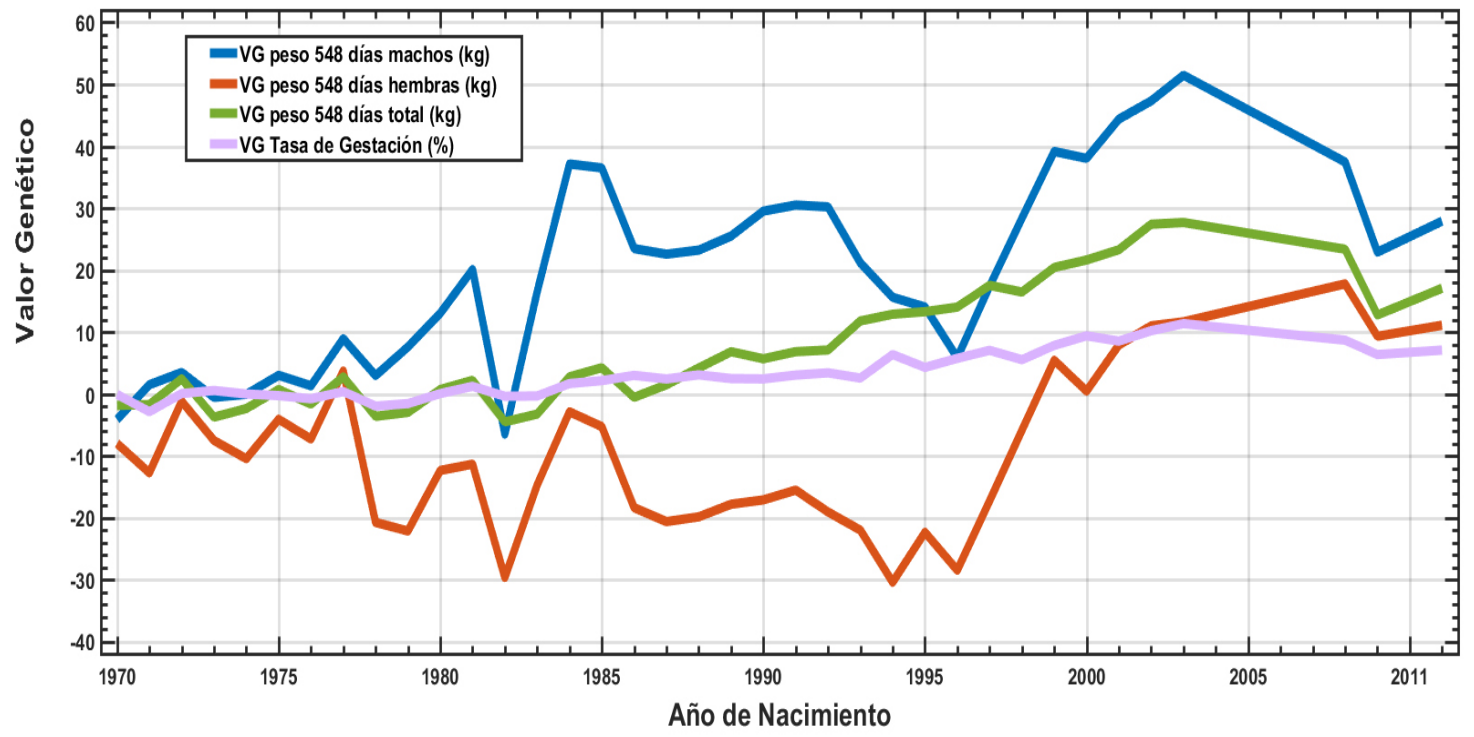


Los valores de $\mathrm{h}^{2}$ para PA548 coinciden con varias referencias en este tipo de animales publicados en Venezuela ${ }^{(19,20)}$, así como con los publicados en ganado B. indicus en diferentes países de América Latina ${ }^{(21)}$. El progreso genético para PA548, fue inferior a lo publicado por otros autores ${ }^{(20,22)}$.

Los estimados de $\mathrm{h}^{2}$ con MU para TG fueron bajos, lo cual es coherente con la mayor parte de las publicaciones sobre caracteres reproductivos ${ }^{(10,23)}$. Sin embargo, los niveles de $\mathrm{h}^{2}$ para TG con MT aumentaron $\left(\mathrm{h}^{2}=0.109\right.$ vs 0.087$)$, incrementándose la precisión promedio de los VG en $15.7 \%$. Los niveles de $r_{\mathrm{g}}$ entre ambos rasgos sugieren ausencia de antagonismo, de manera que un proceso de selección por PV y TG pueden llevarse a la práctica, enfoque ya sugerido en otras publicaciones ${ }^{(11,12,24)}$. La mayor variabilidad genética (Figura 2) y diferentes niveles de $\mathrm{h}^{2}$ y $\mathrm{r}_{\mathrm{g}}$ para PA548 a lo largo de la trayectoria de la edad-sexo (Figura 1) indican que la manifestación de este rasgo no debe considerase como expresión del mismo carácter en ambos sexos. Lo anterior coincide con otros resultados publicados ${ }^{(7,8)}$.

El dimorfismo sexual (DS) muy evidente en estos datos se ha estudiado en detalle en el contexto evolutivo de las poblaciones, existiendo debate sobre la importancia de la heterogeneidad de varianzas entre sexos y sus efectos sobre la especialización y adaptabilidad de las poblaciones ${ }^{(25)}$, mientras que existen planteamientos previos sobre cambios en DS como respuesta correlacionada a la selección por fertilidad. Relacionado con este último punto de vista, los resultados de este trabajo permiten presentar un enfoque novedoso ya que disponemos de los VG en cada sexo para PA548M y PA548H (resultados de la solución del modelo seis, bloque dos), de manera que se puede estimar un DS de origen genético como DSg= VGPA548M - VGPA548H y estos estimados de Dsg se pueden relacionar con los VG de los mismos animales para TG (solución del modelo MT del bloque 0 ). Los resultados del análisis indicaron que una ecuación cuadrática $\left(\mathrm{TG}=0.574+0.1045 * \mathrm{DSg}+0.000765 * \mathrm{DSg}^{2}\right.$ - $0.0000244 * \mathrm{DSg}^{3}$ y $\mathrm{R}^{2}=96.1 \%$ ) fue la de mejor ajuste a los datos, manifestándose un incremento del orden de $+1.2 \%$ en TG por cada $10 \mathrm{~kg}$ de DSg, con un punto máximo cuando $40>\mathrm{DSg}<60$ con $\mathrm{TG}=+4.4 \%$. Por el contrario, cuando $-10>\mathrm{DSg}<0$, la TG fue de $-1.4 \%$. Estos resultados son alentadores, pero se requieren mayores investigaciones sobre esta temática que pueden tener una importante aplicación práctica en los sistemas de producción del ganado de carne.

En este trabajo se detecta una amplia variabilidad genética tanto en PV como en TG de animales del tipo Brahman. Se sugiere utilizar modelos MT, que posibilita incrementos sustanciales en los valores de $\mathrm{h}^{2}$ y en la precisión de los VG estimados, particularmente en TG. El análisis de RA ha indicado que tanto los niveles de $h^{2}$ como de las $r_{g}$ entre el PV de hembras y machos varían a lo largo de la trayectoria de la edad, de manera que no se deben considerar como expresiones del mismo rasgo. Finalmente, y como tendencias adicionales, se ha podido identificar una importante variabilidad genética en dimorfismo sexual, la cual está relacionada con la TG, aunque esta sugerencia requiere de otras investigaciones con mayor número de animales. 


\section{Literatura citada:}

1. Plasse D, Verde O, Arango J, Camaripano L, Fossi H, Romero R, Rodriguez C, Rumbos J. (Co)variance components, genetic parameters and annual trends for calf weights in a Brahman herd kept on floodable savanna. Genet Mol Res 2002;1(4):282-297.

2. Menéndez BA, Guerra D, Planas T, Ramos F, Fernández L. Parámetros genéticos del peso vivo de machos Cebú en prueba de comportamiento en condiciones de pastoreo de Cuba, mediante modelo animal univariado, multicaracteres y regresiones aleatorias. Rev Cubana Cienc Agric 2006;40(4):397-408.

3. Martínez C, Elzo M, Manrique C, Jiménez A. Genetic parameters and breeding values for live weight using random regression models in a Bos taurus-Bos indicus multibreed cattle population in Colombia. Rev Colomb Cienc Pecu 2012;25:548-565.

4. Nobre P, Misztal I, Tsuruta S, Bertrand J, Silva L, Lopes P. Analyses of growth curves of Nellore cattle by multiple-trait and random regression models. J Anim Sci 2003;81:918-926.

5. Lopes F, Ulhôa C, Paulini F, Corrêa M, Sayuri E, Barbosa R. Analysis of longitudinal data of Nellore cattle from performance test at pasture using random regression model. Springer Plus 2012;1:49.

6. Garrick D, Pollak E, Quaas R, Van-Vleck L. Variance heterogeneity in direct and maternal weight traits by sex and percent purebred for Simmental-sired calves. J Anim Sci 1989;67:2515-2528.

7. Rodriguez AF, Van-Vleck L, Cundiff L, Kachman S. Heterogeneity of variance by sire breed, sex, and dam breed in 200- and 365-day weights of beef cattle from a top cross experiment. J Anim Sci 1995;73:2579-2588.

8. Pegolo N, Albuquerque L, Lobo R, Oliveira H. Effects of sex and age on genotype $\times$ environment interaction for beef cattle body weight studied using reaction norm models. J Anim Sci 2011;89:3410-3425.

9. Donoghue K. Genetic evaluation of female reproductive performance. http://www.bifconference.com/bif2002/Baker_Essay_pdfs/Donoghue_02BIF.pdf(02/0 4/2006).

10. Cammack K, Thomas M, ENNS R. Review: Reproductive traits and their heritabilities in beef cattle. Prof Anim Sci 2009;25:517-528.

11. Berry D, Evans R. Genetics of reproductive performance in seasonal calving beef cows and its association with performance traits. J Anim Sci 2014;92:1412-1422. 
12. Mercadante M, Packer I, Razook A, Cyrillo J, Figueiredo L. Direct and correlated responses to selection for yearling weight on reproductive performance of Nelore cows. J Anim Sci 2003;81:376-3841.

13. Guerra D, González D, Rodríguez M, Ramos F. Relación entre el crecimiento y la reproducción en el ganado Cebú cubano. Ciencia y Tecnología Ganadera 2008;2 (3):147-152.

14. Ewel J, Madriz A. Zonas de vida de Venezuela. Ministerio de Agricultura y Cría. Dirección de Investigación. 1968.

15. SAS. SAS/STAT User's Guide. Release 9.0. SAS. Inst. Inc., Cary, NC, USA. 2002.

16. Gilmour A, Gogel B, Cullis B, Thompson R. ASReml User Guide Release 3.0. VSN International Ltd, Hemel Hempstead, HP1 1ES, UK. 2009.

17. Falconer D, McKay F. Introduction to quantitative genetics. 4th ed. Burnt Mill, England. 1996.

18. Jamrozik J, Schaeffer L. Estimates of genetic parameters for a test day model with random regression for production of first lactation. J Dairy Sci 1997;80:762-770.

19. Plasse, D, Verde O, Fossi H, Romero R, Hoogesteijn R, Bastidas P, Bastardo J. (Co)variance components, genetic parameters and annual trends for calf weights in a pedigree Brahman herd under selection for three decades. J Anim Breed Genet 2002; 119:141-153.

20. Arias M, Romero R, Camaripano L, Arriaga L. Genetic and non-genetic parameters for growth traits in a registered Brahman herd. Rev Fac Cs Vet UCV 2013;54(2):78-88.

21. Giannotti J, Packer I, Zerlotti M. Meta-Análise para estimativas de herdabilidade para características de crescimento em bovinos de corte. Rev Bras Zootec 2005;34 (4):11731180.

22. Seprocebu, (http://www.seprocebu.com/GENETICO/. Consultado Sep 15, 2017.

23. Donoghue K. Genetic evaluation of female reproductive performance. Beef Improvement Federation (BIF) annual conference. July 10-13, 2002, in Omaha, Neb., USA. http://www.bifconference.com/bif2002/. Consultado 24 Abr, 2011.

24. Meyer K, Hammond K, Mackjnnon M, Pamel P. Estimates of covariances between reproduction and growth in Australian beef cattle. J Anim Sci 1991;69:3533-3543. 
25. Poissant J, Wilson A, Coltman D. Sex-specific genetic variance and the evolution of sexual dimorphism: A systematic review of cross-sex genetic correlations. Evolution. 2010;64(1):97-107. 